\title{
Association of newly diagnosed type 1 diabetes and autoimmune pancreatitis
}

\author{
Laila Ennazk, Ghizlane El Mghari and Nawal El Ansari \\ Department of Endocrinology, Diabetology and Metabolic Diseases, Mohammed VI University Hospital \\ of Marrakech, Caddi Ayyad University, Marrakech, Morocco
}

Correspondence should be addressed to L Ennazk

Email

lennazk@gmail.com

\section{Summary}

Autoimmune pancreatitis is a new nosological entity in which a lymphocytic infiltration of the exocrine pancreas is involved. The concomitant onset of autoimmune pancreatitis and type 1 diabetes has been recently described suggesting a unique immune disturbance that compromises the pancreatic endocrine and exocrine functions. We report a case of type 1 diabetes onset associated with an autoimmune pancreatitis in a young patient who seemed to present a type 2 autoimmune polyglandular syndrome. This rare association offers the opportunity to better understand pancreatic autoimmune disorders in type 1 diabetes.

\section{Learning points:}

- The case makes it possible to understand the possibility of a simultaneous disturbance of the endocrine and exocrine function of the same organ by one autoimmune process.

- The diagnosis of type 1 diabetes should make practitioner seek other autoimmune diseases. It is recommended to screen for autoimmune thyroiditis and celiac diseases. We draw attention to consider the autoimmune origin of a pancreatitis associated to type 1 diabetes.

- Autoimmune pancreatitis is a novel rare entity that should be known as it is part of the lgG4-related disease spectrum.

\section{Background}

Polyglandular autoimmune syndromes represent a heterogeneous group of rare diseases characterized by an autoimmune process affecting at least two endocrine tissues, sometimes with one or more non-associated endocrine diseases. The discovery of new auto-antigenic targets in neuroendocrine immunology allowed the consideration of new autoimmune diseases. Therefore, new entities are recognized as part of the polyglandular autoimmune syndromes. The diagnosis of autoimmune pancreatitis lacks specific diagnostic criterion. Type 1 diabetes associated with autoimmune pancreatitis is increasingly described in the literature. We report a case of this association.

\section{Case presentation}

A 22-year-old woman, with no family history of autoimmune disorders, was admitted in intensive care for acute epigastric pain and vomiting. She had an impaired general condition and weight loss of $10 \mathrm{~kg}$ in 2 weeks. On examination, Glasgow Coma Scale was at $13 / 15$. Blood pressure was at $120 / 80 \mathrm{mmHg}$ and heart rate at $128 / \mathrm{min}$. Capillary blood glucose was $4 \mathrm{~g} / \mathrm{L}(22.2 \mathrm{mmol} / \mathrm{L})$ with glucosuria and acetonuria in urine examination. Abdominal examination found an epigastric tenderness. The neck examination revealed a homogeneous small goiter without lymphadenopathies.

\section{Investigation}

Laboratory tests showed an inflammatory syndrome with leukocytosis at 22400/mL (NR: 4000-10000/mL). High lipase over ten times the upper limit of normal: 787.9 U/L (NR: 13-60). Creatininemia was at $4 \mathrm{mg} / \mathrm{L}$ (NR: 6-13), urea at $0.21 \mathrm{~g} / \mathrm{L}(0.15-0.45)$. Blood glucose 
was $4.64 \mathrm{~g} / \mathrm{L}(24.75 \mathrm{mmol} / \mathrm{L})$ with sodium at $129 \mathrm{meq} / \mathrm{L}$ (NR: 135-145), hypokalemia at $2.9 \mathrm{meq} / \mathrm{L}$ (NR: $3.5-4.5)$ and a severe acidosis with alkaline reserves at $7 \mathrm{mmol} / \mathrm{L}$ (NR: 22-29). HbA1c was at 11\%. Liver function tests, cholesterol, triglycerides and hepatitis serology were normal. The hormonal balance sheet showed: TSH at $0.05 \mathrm{IU} / \mathrm{mL}$ (NR: $0.25-5$ ) and FT4 at $42.52 \mathrm{pmol} / \mathrm{L}$ (NR: 10.6-19.4). Cortisol at $0800 \mathrm{~h}$ was at $19.43 \mu \mathrm{g} / \mathrm{dL}$ (NR: 8.7-22.40). Electrocardiogram showed a sinus tachycardia without arrhythmias. Autoimmune screen showed positive anti-nuclear antibodies (titre: 1/160), negative anti-mitochondrial and anti-smooth muscle antibodies, positive thyroid peroxidase antibody (titre: $288.32 \mathrm{IU} / \mathrm{mL}$ ) and negative thyrotropin receptor antibodies. Glutamate decarboxylase antibodies were positive. Anti-transglutaminase antibodies were negative. IgG4 in serum were at $2.190 \mathrm{~g} / \mathrm{L}$ (NR: 0.040-0.870). Cervical ultrasonography showed a hypoechogenic gland with increased blood flow.

\section{Treatment}

Treatment consisted of correction of diabetic ketoacidosis by intravenous hydration in addition to a continuous insulin infusion. Symptomatic treatment of hyperthyroidism was established based on steroids and propranolol. A digestive rest was prescribed for the first five days, after which she was on multiple daily insulin injections.

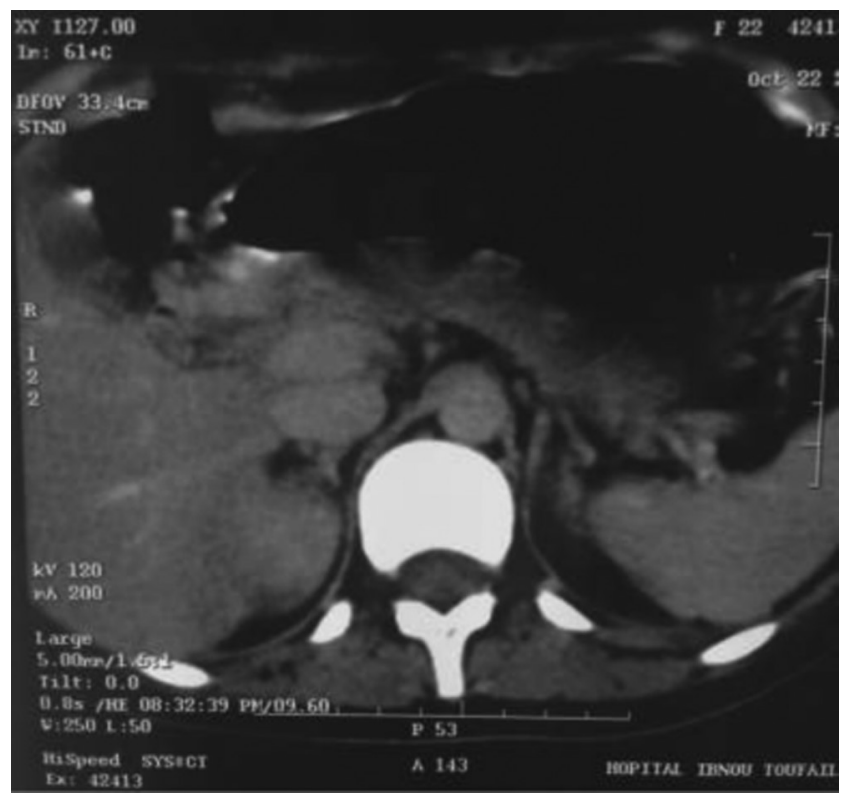

Figure 1

CT scan showing an acute pancreatitis.

\section{Outcome and follow-up}

Clinical evolution was marked by regression of epigastric pain, the tachycardia and tachypnea. The abdominal CT scan at $72 \mathrm{~h}$ showed an acute pancreatitis with swelling pancreas without necrosis (Fig. 1). Free T4 was at normal range within three weeks. At three months, fasting blood glucose was at $1.9 \mathrm{~g} / \mathrm{L}$ and an HbA1c was at $8 \%$.

\section{Discussion}

Autoimmune pancreatitis is a rare entity. It represents $2 \%$ of chronic pancreatic damage (1). Although the concept has been known for decades, it is facing a difficulty in validating diagnostic criteria, and because of the various clinical and serological presentations.

Histologically, the pancreas presents with atrophy of the acini and lymphoplasmacytic infiltration. The most suitable terminology for these histological findings is 'lymphoplasmacytic pancreatitis' (2). Later, increased total immunoglobulin $G$ level has been shown to be associated with pancreatic autoimmune disease, which improves under corticosteroid therapy (2).

More recently, a new classification divides autoimmune pancreatitis into two entities. Type 1 is part of IgG4 sclerosing disease in which IgG4 is the cause of multiple organ damage. Type 2 is described in Western countries as corresponding to acute and benign pancreatic damage, with normal levels of serum immunoglobulins and usually associated with chronic inflammatory bowel disease $(3,4)$.

Serologically, other markers of pancreatic autoimmunity have been studied, including antilactoferrin antibodies, anti-carbonic anhydrase antibody and anti-amylase antibody $\alpha 2 \mathrm{~A}(5)$.

Our patient had a DKA associated with elevated serum lipase. In fact, significant but nonspecific elevation of serum lipase can be seen in DKA. However, the patient seemed to more likely present with acute pancreatitis as the CT scan was consistent with concomitant pancreatic inflammation.

As the concept of autoimmune pancreatitis has changed, the diagnostic criteria has also evolved. The Asian diagnostic criteria (6) requires two criterions: imaging pancreatic abnormalities and serological abnormalities to determine whether high serum IgG4 is present or for the detection of antibodies. This new guidance tries to propose alternative criteria so as to avoid biopsy of an inaccessible organ. In addition, the improvement under corticosteroid treatment noted in our patient is another argument for 
the diagnosis of autoimmune pancreatitis. However, the diagnosis of fulminant type 1 diabetes and concomitant autoimmune pancreatitis could not be made in our patient because of the high level of $\mathrm{HbA1c}$ at diagnosis.

Simultaneous autoimmune pancreatitis and type 1 diabetes is not well known. Only two cases have been reported in the literature by the same Japanese author (7, 8 ). He suggests to talk about 'the exocrine and endocrine pancreatic autoimmune damage'. Cell-mediated autoimmunity explains the co-existence of autoimmune diabetes and autoimmune pancreatitis. It is found that among type 1 diabetics, those carrying HLA DRB1 0405/ DQB1 0401 are most at risk. Indeed, 88\% of type 1 diabetics express the anti-amylase $\alpha 2 \mathrm{~A}$, which may have a role in assessing the risk of autoimmune pancreatitis in type 1 diabetes (5). This combination opens up future prospects for screening the risk of pancreatitis in type 1 diabetics.

\section{Declaration of interest}

The authors declare that there is no conflict of interest that could be perceived as prejudicing the impartiality of the research reported.

\section{Funding}

This research did not receive any specific grant from any funding agency in the public, commercial or not-for-profit sector.

\section{Patient consent}

Written informed consent was obtained from the patient/patient's mother for publication of this case report.

\section{References}

1 Okasaki K \& Chiba T 2002 Autoimmune related pancreatitis. Gut 5 1-4. (doi:10.1136/gut.51.1.1)

2 Yoshida K1, Toki F, Takeuchi T, Watanabe S, Shiratori K \& Hayashi N 1995 Chronic pancreatitis caused by an autoimmune abnormality. Proposal of the concept of autoimmune pancreatitis. Digestive Diseases and Sciences 40 1561-1568. (doi:10.1007/BF02285209)

3 Notohara K1, Burgart LJ, Yadav D, Chari S \& Smyrk TC 2003 Idiopathic chronic pancreatitis with periductal lymphoplasmacytic infiltration: clinicopathologic features of 35 cases. American Journal of Surgical Pathology 27 1119-1127. (doi:10.1097/00000478-20030800000009)

4 Chari ST, Smyrk TC, Levy MJ, Topazian MD, Takahashi N, Zhang L, Clain JE, Pearson RK, Petersen BT, Vege SS, et al. 2006 Diagnosis of autoimmune pancreatitis: the Mayo Clinic experience. Clinical Gastroenterology and Hepatology 4 1010-1016. (doi:10.1016/j. cgh.2006.05.017)

5 Endo T, Takizawa S, Tanaka S, Takahashi M, Fujii H, Kamisawa T \& Kobayashi T 2009 Amylase alpha-2A autoantibodies: novel marker of autoimmune pancreatitis and fulminant type 1 diabetes. Diabetes 58 732-737. (doi:10.2337/db08-0493)

6 Otsuki M, Chung JB, Okazaki K, Kim MH, Kamisawa T, Kawa S, Park SW, Shimosegawa T, Lee K, Ito T, et al. 2008 Asian diagnostic criteria for autoimmune pancreatitis: consensus of the Japan-Korea Symposium on Autoimmune Pancreatitis. Journal of Gastroenterology 43 403-408. (doi:10.1007/s00535-008-2205-6)

7 Taniguchi T, Seko S, Okamoto M, Hamasaki A, Ueno H, Inoue F, Nishida O, Miyake N \& Mizumoto T 2000 Association of autoimmune pancreatitis and type 1 diabetes. Diabetes Care 23 1592-1594. (doi:10.2337/diacare.23.10.1592)

8 Taniguchi T, Tanaka J, Seko S, Okazaki K \& Okamoto M 2001 Association of rapid-onset type 1 diabetes and clinical acute pancreatitis positive for autoantibodies to the exocrine pancreas. Diabetes Care 24 2156-2157. (doi:10.2337/diacare.24.12.2156)

Received in final form 7 September 2016 Accepted 14 September 2016 